\title{
On a Symmetry Argument for the Guidance Equation in Bohmian Mechanics*
}

\author{
Bradford Skow
}

\begin{abstract}
Bohmian mechanics faces an underdetermination problem: when it comes to solving the measurement problem, alternatives to the Bohmian guidance equation work just as well as the official guidance equation. Dürr, Goldstein, and Zanghì have argued that of the candidate guidance equations, the official guidance equation is the simplest Galilean-invariant candidate. This symmetry argument-if it worked—would solve the underdetermination problem. But the argument does not work. It fails because it rests on assumptions about how Galilean transformations (especially boosts) act on the wavefunction that are (in this context) unwarranted. My discussion has larger morals about the physical significance of certain mathematical results (like, for example, Wigner's theorem) in non-orthodox interpretations of quantum mechanics.
\end{abstract}

\section{Introduction}

Bohmian mechanics is a proposed replacement for orthodox quantum mechanics. It solves the measurement problem, the main problem orthodox quantum mechanics faces. But there are still questions about Bohmian mechanics, and in this paper I want to discuss one of them.

While in orthodox quantum mechanics particles never have definite locations in space, in Bohmian mechanics they always do. So in addition to Schrödinger's

*Published in International Studies in the Philosophy of Science 24 (2010): 393410. 
equation, Bohmian mechanics includes a new equation, the guidance equation, that governs how the particles move. Now, to explain why Bohmian mechanics solves the measurement problem one must use the guidance equation. But the explanation does not require that the guidance equation be exactly as it is. There are other 'candidate guidance equations' that work just as well as the official guidance equation when it comes to solving the measurement problem. And that means that there are other Bohm-like theories, incorporating different candidate guidance equations, that solve the measurement problem in the same way Bohmian mechanics does. So the question I am interested in is: insofar as we are interested in solving the measurement problem, should we have a higher degree of belief in Bohmian mechanics than in its rivals? Or should we think they are all equally good? This is the underdetermination problem for Bohmian mechanics.

Abstractly speaking, one way to solve the underdetermination problem for Bohmian mechanics is to argue that the official guidance equation has some 'theoretical virtue' that its alternatives lack, and that theories having that virtue are (other things being equal) to be preferred to their rivals. And there is an argument out there identifying such a virtue. It is an argument that, among the candidate guidance equations, the official guidance equation is the simplest Galilean-invariant candidate (see Dürr, Goldstein, and Zanghì 1992; 1996).

My aim in this paper is to argue that the symmetry argument for the guidance equation cannot be used as an argument for preferring Bohmian mechanics to its rivals in this context. Briefly, my objection to the symmetry argument is that it makes unjustified assumptions about how Galilean transformations act on the wavefunction. But I want to stress at the outset that I only object to the use of the symmetry argument in one particular context. I have no objection to the use of the symmetry argument in other contexts. For example, I certainly have no objection to a use of the symmetry argument in 'the context of discovery': the symmetry argument is an excellent line of thought for physicists to have had when they were trying to find some equation to play the role of the guidance equation.

For all I say in this paper, there may be other arguments for preferring the guidance equation to its alternatives that do succeed in this context. (See, for example, Peruzzi and Rimini 2000 for one such argument.) So why do I focus so 
much attention on the symmetry argument? I have two reasons. First, symmetry arguments are a form of argument that we see all over physics (and philosophy too). It is the kind of argument we might most expect to work. That it does not work in this case is something of a surprise. Second, I think that the failure of the symmetry argument can teach us something about the significance that some well-known theorems in the mathematical foundations of quantum mechanics have in Bohmian mechanics.

\section{Background to the Problem}

I begin with a brief review of Bohmian mechanics. In elementary orthodox quantum mechanics, the physical state of a system (a collection of particles) at each time is represented by a (unit-length) vector in a Hilbert space-the wavefunction. There are two dynamical laws that govern the evolution of the wavefunction. Unless a measurement occurs, the wavefunction evolves in accord with Schrödinger's equation; when a measurement occurs, the wavefunction collapses so that the system has a definite value for the property being measured.

Bohmian mechanics is an alternative to orthodox quantum mechanics. Some of the formal apparatus is the same. Bohmian mechanics still makes use of the wavefunction (a mathematical object). But in Bohmian mechanics the wavefunction always evolves in accord with Schrödinger's equation. The wavefunction never collapses. In addition, the wavefunction plays a different representational role in the theory. It does not represent the physical state of the particles. Instead, the physical state of the particles is given by a point in configuration space. (So what does the wavefunction represent? More on that below.)

Let's look at the simplest example: a single particle without spin moving in one spatial dimension. Then configuration space is just $\mathbb{R}$. The wavefunction over time is given by a function $\psi: \mathbb{R} \times \mathbb{R} \rightarrow \mathbb{C}$ where for each $t$ the function $\psi(x, t)$ is (measurable and) square-integrable. And the particle's position over time is given by a (differentiable) function $\gamma: \mathbb{R} \rightarrow \mathbb{R}$.

Schrödinger's equation governs the evolution of the wavefunction. The new law describing the evolution of the particle's position is the guidance equation. The 
guidance equation gives the particle's velocity at $t$ as a function of the wavefunction at $t$, its spatial derivative at $t$, and the particle's position at $t:^{1}$

$$
\gamma^{\prime}(t)=\frac{\hbar}{m} \operatorname{Im}\left[\frac{(\partial \psi / \partial x)(\gamma(t), t)}{\psi(\gamma(t), t)}\right] \text {. }
$$

Now let us see how the underdetermination problem arises in Bohmian mechanics. Bohmian mechanics matches the predictions the orthodox theory makes about the outcomes of positions measurements as follows. ${ }^{2}$ Consider the well-known two-slit experiment: electrons are fired toward a barrier with two slits in it, and are detected at a screen behind the barrier. Each time the experiment is performed the initial wavefunction of the emitted electron is the same. The orthodox theory says that the probability that the electron will be detected in a given (measurable) region $R$ on the screen is equal to $\int_{R}|\psi(x, t)|^{2}$ (where $t$ is the time at which the electron arrives at the screen) - this even though (the orthodox theory also says that) during the experiment, the electron had no definite position (and so did not definitely pass through the top slit, and did not definitely pass through the bottom slit).

The story Bohmian mechanics tells about this experiment is very different. That theory says that the electron had a definite location at each time, and either passed through the top slit, or through the bottom slit. The theory is deterministic: which point on the screen the electron hits is determined by the initial wavefunction

\footnotetext{
${ }^{1}$ This is the form of the guidance equation preferred by (among others) Bell (1987b) and Dürr, Goldstein, and Zanghì (1992). There are alternative, mathematically equivalent forms of the equation-Bohm 1952, for example, prefers to write it as a second-order differential equation similar in form to Newton's second law. Although these forms of the guidance equation are mathematically equivalent there may be conceptual differences between them, and these differences may suggest different strategies for justifying the equation. But in this paper I will focus only on the first-order formulation of the equation.

${ }^{2}$ As is well known, because the word 'measurement' appears in the formulation of orthodox theory it is not clear just what predictions the theory does make. (The word 'measurement' does not appear in the formulation of Bohmian mechanics.) So it is not clear just what it takes for an interpretation of (or replacement for, or completion of) orthodox quantum mechanics to match that theory's predictions. I am going to set this problem aside.
} 
and initial position of the electron. The electron does not hit the same point on the screen on every run of the experiment because the electron's initial location differs on different runs of the experiment (even though the initial wavefunction does not). The frequency with which the particle begins in some region $S$ near the source is equal to $\int_{S}\left|\psi\left(x, t_{0}\right)\right|^{2}$ ( $t_{0}$ is the initial time). Using the guidance equation, it can be shown that the frequency with which electrons will be found in a region $R$ near the screen is then equal to $\int_{R}|\psi(x, t)|^{2}$. (On some views about probability in Bohmian mechanics, what is shown is actually that the probability is very close to 1 that after many runs the frequency is close to $|\psi(x, t)|^{2}$.) These frequencies, then, match the probabilities given by the orthodox theory. ${ }^{3}$

In general, the guidance equation can be used to show that the particle dynamics 'preserve probability': roughly speaking, the guidance equation ensures that if the frequency with which particles with wavefunction $\psi$ are to be found in $R$ at some time $t_{0}$ is given by $\int_{R}\left|\psi\left(x, t_{0}\right)\right|^{2}$, then the frequency with which they are to be found in $R$ at any other time $t$ is also given by $\int_{R}|\psi(x, t)|^{2}$. The fact that the guidance equation can be used to show this is the main role it plays in justifying the claim that Bohmian mechanics matches the predictions of orthodox quantum mechanics.

We can now see just what kind of underdetermination problem Bohmian mechanics faces. There are alternatives to the guidance equation that also 'preserves probability'. So there are alternatives to Bohmian mechanics which are just as successful at solving the measurement problem: on these alternatives particles also always have definite locations, and the frequencies also match the probabilities given

${ }^{3}$ This sketch of the Bohmian solution to the measurement problem resembles the one given in Bell 1987a. But the full story of how Bohmian mechanics solves the measurement problem is much more complicated. For example, I ignore the fact that due to the pervasiveness of quantum entanglement particles almost never have their own wavefunctions. Bohmians must explain why we are entitled to treat them as if they do. I also say nothing about why Bohmians are entitled to assume that the frequency with which electrons appear in some region $S$ near the source is given by $\int_{S}|\psi|^{2}$. For the full story, including how the theory deals with measurements of things other than position, see Dürr, Goldstein, and Zanghì 1992. Although what I say is oversimplified in many ways, it is accurate enough to let us see the role the guidance equation plays. 
by the orthodox theory. ${ }^{4}$ Since all of these alternatives predict the same frequencies, they are empirically equivalent. ${ }^{5}$

So even if we think that the Bohmian strategy for solving the measurement problem is the best strategy, we will not yet have singled out just what theory to accept. Should we accept Bohmian mechanics, or some other Bohm-like theory that contains an alternative to the guidance equation?

It is true that in some contexts, this question does not appear very pressing. If what has us worried is whether there is any theory that solves the measurement problem, we won't be upset to have found many similar theories that solve it. Nevertheless, in other contexts, it is a pressing question. Physicists who want to extend the Bohmian strategy to more sophisticated quantum theories need to know whether they should be trying to extend Bohmian mechanics or one of its alternatives. And, physics aside, it is an interesting philosophical question whether we have here a genuine example of a situation in which the empirical evidence alone does not favour a theory over one of its rivals.

${ }^{4}$ In slightly more detail, to say that the guidance equation preserves probability is to say that

$$
\frac{\partial \rho}{\partial t}(x, t)+\nabla(\rho(x, t) v(x, t))=0,
$$

where $\rho(x, t)=|\psi(x, t)|^{2}$ and $v(x, t)$ is the velocity field given by the guidance equation. Inspection shows that if we changed the guidance equation so that

$$
\tilde{v}(x, t)=v(x, t)+\frac{w(x, t)}{|\psi|^{2}}
$$

where $v$ is a solution to the original guidance equation and $w(x, t)$ is divergencefree, then the continuity equation is still true of $\tilde{v}$. So the guidance equation is not the unique equation that, together with $|\psi|^{2}$, satisfies the continuity equation; it is not the unique equation that 'preserves probability'. (An extended discussion of alternative Bohm-like theories may be found in Deotto and Ghirardi 1998.)

${ }^{5}$ They are not empirically equivalent if we give up the assumption that the frequency with which electrons begin in some region $S$ near the source is given by $\int_{S}|\psi|^{2}$ - that is, if we give up the quantum equilibrium hypothesis. But I am looking for a justification of the guidance equation that holds even under this hypothesis. 


\section{Symmetry Arguments for Dynamical Laws}

To understand the symmetry argument for the guidance equation it will help to look at a simpler symmetry argument for a different dynamical law. In this simpler setting it will be easier to identify the structure of this kind of argument.

The law I will look at is Newton's first law of motion, the law of inertia: a free body (one not acted on by any forces) does not accelerate. Before looking at the symmetry argument for this law let me say something about the status of this law in Newtonian mechanics.

The law of inertia is a consequence of Newton's second law, ${ }^{6}$ so from one point of view it is redundant to include both. Why state the first law as a separate law, instead of justifying it by deriving it from the second law?

The first law does merit a separate statement when we think about the causes of motion in a certain way. As Aristotle did, divide motions into 'natural' motions and 'forced' or 'violent' motions. A body's natural motion is the way it will move when it is not interfered with. If we think about motion in this way, we will want two laws of motion: one law specifying bodies' natural motions, and the other specifying how their states of motion deviate from their natural motion when they are interfered with. This is the structure we see in Newton's first two laws (the first law specifies the natural motions).

In fact, if we think about the structure of Newton's laws in this way, then we might seek a justification for them (and the law of inertia in particular) to address the same kind of worries about underdetermination that Bohmian mechanics faces. If we take seriously the distinction between forced and natural motions, then the actual motions of material bodies appear to underdetermine what their natural motions are. For there appear to be countless ways to divide their actual motions into the component due to natural motion and the component due to interference. We might put it this way: a generalized version of Newton's second law says that $(F+m I)=$

\footnotetext{
${ }^{6}$ Whether the first law follows from the second law by setting $F=0$ is a matter of dispute (see, for example, Earman and Friedman 1973; an interpretation of the first law that makes it a consequence of the second appears on page 337). But since I am using Newton's laws just to illustrate the type of symmetry argument I am interested in, it is safe to assume that this understanding of the first law is correct.
} 
$m a$, where $I$ gives the body's 'natural' acceleration, and $F / m$ gives its acceleration due to external forces $F$. A generalized law of inertia, then, says that for free bodies $a=I$. With suitable adjustments in the force laws, laws with different values for $I$ are all empirically equivalent (and all equivalent to Newton's law, which sets $I=0)^{7}$

So what is the symmetry argument for the first law? In outline it goes like this. The argument starts by assuming that the correct spacetime setting for the laws we are looking for is Galilean spacetime. ${ }^{8}$ Now the laws must be invariant under the symmetries of spacetime. And it can be proved that Newton's law of inertia is the only candidate law governing the motion of free bodies that is invariant under the symmetries of Galilean spacetime. That is why Newton's law is the correct law-there are no alternatives.

Let's go through the proof that only Newton's law is invariant under Galilean transformations. Let $\gamma: \mathbb{R} \rightarrow \mathbb{R}^{3}$ be the function that gives the position of some free body as a function of time, relative to some inertial frame of reference. I assume that $\gamma$ is differentiable (which entails that the body does not move discontinuously) and defined on all of $\mathbb{R}$ (the particle does not pop in and out of existence). Since how a free body behaves does not depend on what other things there are (I assume), we can suppose that the free body is alone in the universe. A law governing the motion of free bodies says how a free body's velocity changes with time. What is it for such a law to be invariant under Galilean transformations-the symmetries of Galilean spacetime? A symmetry of spacetime is just a function from spacetime to itself that preserves the geometrical structure of spacetime. Corresponding to each symmetry of spacetime is a transformation of the state (in this case the position) of the particle at each time. In this case the correspondence is obvious: a spatial translation $L$ given by $(t, \mathbf{x}) \mapsto(t, \mathbf{x}+\alpha)$ (where $\alpha \in \mathbb{R}^{3}$ is a fixed vector) transforms

\footnotetext{
${ }^{7} \mathrm{I}$ am discussing this example as an aid to understanding the structure of the symmetry argument for the guidance equation. So do not think that I take this motivation for seeking a justification for the law of inertia seriously. It may be that we should reject the presupposition that there is a distinction to be made between forced motion and natural motion.

${ }^{8}$ Let's not worry about how we could have grounds for this assumption in a context in which we do not know what the correct law of inertia is.
} 
$\gamma$ to $\gamma_{L}$ where $\gamma_{L}(t)=\gamma(t)+\alpha$; a boost $G$ given by $(t, \mathbf{x}) \mapsto(t, \mathbf{x}+t \mathbf{v})$ transforms $\gamma$ to $\gamma_{G}$ where $\gamma_{G}(t)=\gamma(t)+t \mathbf{v}$, and so on. (I belabour this point because when it comes to looking at how Galilean transformations act on the wavefunction, the correspondence will not be so obvious.)

Now we can say what it is for a candidate law of inertia to be invariant under Galilean transformations: it is invariant iff

- $\gamma$ is a solution iff for each Galilean transformation $X, \gamma_{X}$ is also a solution.

Now we can look at the proof that Newton's version of the law of inertia is the only candidate law of inertia that is invariant under Galilean transformations. Here is how the alternatives to Newton's law get ruled out. Any alternative to Newton's law of inertia will say that under some circumstances a free body accelerates. To do this it must specify a direction for the body to accelerate in. How could the law do that? One way is to name a particular direction in space ('galactic north'?) and say that free bodies always accelerate in that direction. But that law is not invariant under spatial rotations. Start with any solution to the law; rotate the solution through any angle less than $2 \pi$ about some axis not parallel to galactic north; after the rotation the particle is not accelerating toward galactic north. Another way is to name a particular point in space (the 'centre of the universe'?) and say that the particle accelerates in the direction that points from the centre of the universe toward the particle's current position. But this method is not invariant under spatial translations. A final way to specify a direction is to use the particle's velocity. But this method is not invariant under boosts. There are no other legitimate methods for specifying a direction for free bodies to accelerate in. So there are no alternatives to Newton's law of inertia that are invariant under the symmetries of Galilean spacetime.

More formally, we assume that the law has the form

$$
\gamma^{\prime \prime}(t)=A\left(\gamma(t), \gamma^{\prime}(t)\right)
$$

That is, we assume that the free body's acceleration is some function $A$ of at most its position and velocity; its acceleration does not depend on any other quantities. (That is, other quantities are definitely ruled out. It will turn out that $A$ does not 
even depend on position and velocity.) Where does this assumption come from? As I said earlier, since it is a free body, one not being interfered with, it makes sense to assume that $A$ does not depend on any other body's position or velocity. But why rule out higher derivatives of $\gamma$ ? This is an interesting question; I do not know the answer. ${ }^{9}$ But for my purposes, it is not important.

Now the argument proceeds: for any spatial translation $L, \gamma_{L}^{\prime \prime}=\gamma^{\prime \prime}$, so the law is Galilean invariant only if

$$
\gamma^{\prime \prime}(t)=A\left(\gamma(t)+\alpha, \gamma^{\prime}(t)\right)
$$

for every $\alpha \in \mathbb{R}^{3}$. So the value of $A$ does not depend on $\gamma(t) ; A$ is a function at most of $\gamma^{\prime}(t)$. Similarly, for any boost $G, \gamma_{G}^{\prime \prime}=\gamma^{\prime \prime}$, so the law is Galilean invariant only if

$$
\gamma^{\prime \prime}(t)=A\left(\gamma^{\prime}(t)+\mathbf{v}\right)
$$

for every $\mathbf{v} \in \mathbb{R}^{3}$. So the value of $A$ does not depend on $\gamma^{\prime}(t)$ either. This means that the law is

$$
\gamma^{\prime \prime}(t)=C,
$$

where $C$ is some constant vector. Then for each spatial rotation $R$ we have

$$
\gamma_{R}^{\prime \prime}(t)=C,
$$

which can happen only if $C=0$ (the only vector that is fixed by all rotations is the 0 vector). And that is what was to be shown.

Now that we have seen the argument, I want to highlight what we needed to assume at the outset before we could give the argument. The following two facts served as 'input' to the argument:

(i) We are seeking a Galilean invariant law.

\footnotetext{
${ }^{9}$ Some equations using higher derivatives can be ruled out; the equation $\gamma^{\prime \prime}=$ $\gamma^{\prime \prime \prime}$ is equivalent to $\gamma^{\prime \prime}=k+\gamma^{\prime}$ where $k$ is some constant, and (as we will see) that equation is not Galilean invariant. I am not sure how far this line of thought extends.
} 
(ii) Translations act on the particle's state by $\gamma(t) \mapsto \gamma(t)+\alpha$, and boosts act on the particle's state by $\gamma(t) \mapsto \gamma(t)+t v$.

The facts listed in (ii) are examples of a more general kind of fact: in general, the argument presupposed facts about how Galilean transformations act on particle states. It is important that these facts are prior to facts about what laws govern particle motions. Only then are facts about how Galilean transformations act on particle states an independent constraint on which candidate law of inertia we accept. For suppose that for each candidate law of inertia we were allowed to choose how Galilean transformations act on states when that law is being considered. Presumably, for each candidate law there is some (maybe extremely weird or counterintuitive) way for Galilean transformations to act on states that renders that candidate 'Galilean invariant'. So if there are no prior facts about which transformations are the correct ones, then the symmetry argument cannot be used to argue against the alternatives to Newton's law.

The same goes for a symmetry argument for the guidance equation. It will require as input facts about how Galilean transformations act on the wavefunction, and those facts need to be independently grounded.

I go on about this not because I think anyone will be tempted to 'save' the Galilean invariance of some strange alternative to Newton's law of inertia by asserting that Galilean transformations do not act on particle states the way we ordinarily think they do. What particles are and how they behave makes it impossible to deny that Galilean transformations act on them in the way we ordinarily think they do. But things are different with the wavefunction in Bohmian mechanics. It is not clear what the wavefunction is or how it behaves in Bohmian mechanics. (At the very least, different Bohmians appear to say different things.)

So far I have been talking about (ii). I also want to say something about (i). Like the symmetry argument for the law of inertia, the symmetry argument for the guidance equation will also start from the claim that we are seeking a Galilean invariant law. The argument will assume that Galilean spacetime is the correct setting for Bohmian mechanics and aim to conclude that (1) is the correct guidance equation. So the argument attempts to use a claim about spacetime structure to justify a claim about the dynamical laws. One might, of course, be interested in constructing 
an argument that runs in the other direction: an argument that starts from the claim that Schrödinger's equation and (1) are the dynamical laws and aims to justify some conclusion about which spacetime geometry is appropriate for Bohmian mechanics. Valentini (1997) develops an argument like this, and reaches the (surprising) conclusion that the appropriate spacetime for a first-order equation like the guidance equation is not Galilean spacetime. Instead (he argues) the appropriate spacetime is Aristotelian spacetime - a spacetime that provides a notion of absolute rest. Clearly this is a very different kind of argument from the symmetry argument I will examine. While both arguments connect dynamics and spacetime symmetries, each takes as given what the other argument aims to justify. Of course, the arguments are not entirely independent. If Valentini's argument is good then the symmetry argument for the guidance equation cannot succeed. For if it did succeed, then an appeal to Galilean spacetime would have justified a dynamical law that does not belong in Galilean spacetime in the first place. But the grounds on which I will object to the symmetry argument are independent of Valentini's claims. My objections apply even if we grant that first-order equations like the guidance equation may belong in Galilean spacetime.

With these preliminaries out of the way, let us see how a symmetry argument for the guidance equation is supposed to go.

\section{A Symmetry Argument for the Guidance Equation?}

Dürr, Goldstein, and Zanghì $(1992,1996)$ want to give an argument for the guidance equation in Bohmian mechanics that resembles the above argument for Newton's law of inertia. It is not perfectly analogous, because they do not purport to rule out all alternatives to the guidance equation. They claim only that the guidance equation is the simplest alternative. ${ }^{10}$

In the argument for Newton's law we started with the restriction that candidate laws should have a free body's acceleration at $t$ depend (at most) only on the free body's own position and velocity at $t$. In the argument for the guidance equation

\footnotetext{
${ }^{10}$ It is not clear to me that Dür et. al. offer the symmetry argument as an argument for preferring Bohmian mechanics to its Bohm-like rivals. But Wallace (2008, section 6.5) sees this as a use to which the argument could be put.
} 
we start with the restriction that candidate laws should have the particle's velocity at $t$ depend (at most) on: the value of the wavefunction and its spatial derivative at $(\gamma(t), t)$, the particle's mass, and $\hbar$. So we want to find a function $F$ so that

$$
\gamma^{\prime}(t)=F\left(\psi(\gamma(t), t), \frac{\partial \psi}{\partial x}(\gamma(t), t), m, \hbar\right),
$$

and we are going to use the requirement that (8) be Galilean invariant (and some other constraints too) to help us find it.

Dürr et al. work in three spatial dimensions and start with the idea that $\gamma^{\prime}(t)$ should be proportional to

$$
\frac{\nabla \psi(\gamma(t), t)}{\psi(\gamma(t), t)} .
$$

The use of $\nabla \psi$ comes from the requirement that $\gamma^{\prime}(t)$ be invariant under rotations, and division by $\psi$ comes from the requirement that changing the wavefunction from $\psi(x, t)$ to $z \psi(x, t)$ (where $z$ is any complex number) should not change $\gamma^{\prime}(t)$. Since to keep things simple I am using one spatial dimension, this corresponds to starting with

$$
\frac{(\partial \psi / \partial x)(\gamma(t), t)}{\psi(\gamma(t), t)} .
$$

While questions might be raised about the justification for starting with (9), I want to focus on the next two appeals to symmetry considerations. I will explain them before criticizing them.

First, for equation (8) to make sense, we need to take either real or imaginary parts of (9). Dürr et. al. consider time reversal to decide which. The time reversal operation $K$ takes $\gamma(t)$ to $\gamma_{K}(t)=\gamma(-t)$, so $\gamma_{K}^{\prime}(t)=-\gamma^{\prime}(-t)$. We need this to happen on the right hand side of equation (8) as well. So what happens to the real and imaginary parts of (9) under time reversal? Well, time reversal acts on the wavefunction by taking $\psi(x, t)$ to $\psi_{K}(x, t)=\psi^{*}(x,-t)$. Under this transformation,

$$
\operatorname{Re}\left[\frac{\left(\partial \psi_{K} / \partial x\right)(\gamma(t), t)}{\psi_{K}(\gamma(t), t)}\right]=\operatorname{Re}\left[\frac{(\partial \psi / \partial x)(\gamma(t),-t)}{\psi(\gamma(t),-t)}\right],
$$


while

$$
\operatorname{Im}\left[\frac{\left(\partial \psi_{K} / \partial x\right)(\gamma(t), t)}{\psi_{K}(\gamma(t), t)}\right]=-\operatorname{Im}\left[\frac{(\partial \psi / \partial x)(\gamma(t),-t)}{\psi(\gamma(t),-t)}\right] .
$$

So we have to choose the latter.

Second, equation (8) must be invariant under boosts. Now a boost that increases velocities by $v$ acts on the wavefunction at $t=0$ by taking $\psi(x, 0)$ to $\psi_{G}(x, 0)=e^{i m v x / \hbar} \psi(x, 0)$ (where $m$ is the mass of the particle). So

$$
\operatorname{Im}\left[\frac{\left(\partial \psi_{G} / \partial x\right)(\gamma(0), 0)}{\psi_{G}(\gamma(0), 0)}\right]=\operatorname{Im}\left[\frac{(\partial \psi / \partial x)(\gamma(0), 0)}{\psi(\gamma(0), 0)}\right]+\frac{m}{\hbar} v
$$

However, we need

$$
\gamma_{G}^{\prime}(0)=\gamma^{\prime}(0)+v
$$

so we multiply by $\hbar / m$ to get (1).

I do not think that this succeeds as a justification of the guidance equation. And that is because I do not think that Dürr et. al. are entitled to the assumptions about how time reversal and boosts act on the wavefunction that are used in the argument. In the next section I explain why.

\section{How do Galilean Transformations Act on the Wavefunction?}

The justification of the guidance equation just presented depends on prior facts about how certain symmetries of Galilean spacetime (boosts and time reversal) act on the wavefunction. The assumptions are:

(A) Time reversal acts on the wavefunction by $\psi(x, t) \mapsto \psi^{*}(x,-t)$.

(B) Boosts act on the wavefunction by $\psi(x, 0) \mapsto e^{i m v x / \hbar} \psi(x, 0)$.

I will start by focusing on (B) (I will have something to say about (A) later). Dürr, Goldstein, and Zanghì assert that $(\mathrm{B})$ is true, but they do not offer any justification for it. I will argue that there is no reason to think that (B) is true in Bohmian mechanics.

(B) is true in the orthodox interpretation of quantum mechanics. But Bohmians cannot tell the story about why (B) is true that the orthodox interpretation tells. 
It is, perhaps, worth going through this in some detail. I will look at two orthodox justifications for (B). Here is the first one:

The first justification. The action of boosts will be represented by a one-parameter group of unitary operators on the Hilbert space in which the wavefunction lives. ${ }^{11}$ But which one-parameter group? We look to classical mechanics for guidance. In Hamiltonian mechanics, the mathematical object representing position is associated with the generator of boosts. The association is made as follows: in Hamiltonian mechanics, the phase space which represents the possible instantaneous states of the particle has a natural symplectic structure. The mathematical object representing position is a real-valued function $f$ on phase space. Using this function one may define the one-form $m d f$, where $m$ is the mass of the particle. The symplectic form on phase space associates a vector field $V$ with this one-form. And $V$ is the infinitesimal generator of boosts-if $x$ represents the actual state of the particle, then the point $v$ units along the integral curve of $V$ through $x$ represents the particle as being in the same place, but moving $v$ units faster.

We use an analogous procedure to identify how boosts are represented by operators on our Hilbert space. In orthodox quantum mechanics, the position operator $Q$ is the self-adjoint operator $\psi \mapsto x \psi$. And there is a natural way to associate a one-parameter group of unitary operators with $Q$. For each $v, \exp (v c i Q)$ is a unitary operator ( $c$ is a real constant). Looking again to the Hamiltonian case, we choose the constant $c$ to be $m / \hbar$.

Now it may be that even a proponent of the orthodox interpretation of quantum mechanics would hesitate to fully endorse this justification. (Some might hesitate to lean too hard on analogies with classical mechanics.) But there are several additional reasons why Bohmians cannot give this justification for (B).

\footnotetext{
${ }^{11}$ And together with the operators that represent spatial translations, rotations, and time translations, they will form a projective representation of the Galilean group. But this further constraint will not matter for what follows.
} 
First, this justification relies on the claim that $Q$ is the self-adjoint operator that represents the position of the particle. But in Bohmian mechanics, position is not represented by $Q$; it is not represented by any self-adjoint operator at all. The particle's position is represented by points in configuration space. So even if Bohmians should think that the operators that represent boosts are somehow associated with the mathematical object that represents position, there is no reason to look for operators associated with $Q$.

Second, this justification presupposes that boosts will be represented by a one-parameter group of unitary operators. Why think that? Even if we assume that boosts will be represented by a one-parameter group of linear operators, why must they be unitary? In the orthodox theory, this question has a straightforward answer: boosts are supposed to be symmetries of the theory, and a symmetry must leave the physically relevant structure of the state space invariant. And in the orthodox theory, the physically relevant structure is (an aspect of) the inner product structure. That structure is physically relevant because of the role it plays in the collapse postulate: if $A$ is a (non-degenerate) self-adjoint operator representing some observable and $\phi$ is a unit-length eigenvector of $A$ with eigenvalue $\lambda$, then the probability that a measurement of $A$ will have the outcome represented by $\lambda$ is $|\langle\psi, \phi\rangle|^{2}$, the square of the length of the projection of $\psi$ onto $\phi$. So the action of any symmetry, and of boosts in particular, must preserve $|\langle\psi, \phi\rangle|$. Wigner's theorem establishes that transformations that preserve $|\langle\psi, \phi\rangle|$ must be linear and unitary (or anti-linear and anti-unitary, but other considerations show that boosts must be unitary). ${ }^{12}$

That is why, in the orthodox theory, boosts must be represented by a oneparameter group of unitary operators. But the physical meaning of the inner product on the space of wavefunctions in Bohmian mechanics is different from its meaning in orthodox quantum mechanics—or, at least, its meaning is arrived at differently. It is true that in Bohmian mechanics the inner product is used to give probabilities for outcomes of position measurements. ${ }^{13}$ But its having this physical significance

\footnotetext{
${ }^{12}$ The fact that boosting by $v$ is equivalent to boosting twice by $v / 2$ can be used to establish that the transformations representing boosts must be unitary.

${ }^{13}$ As above, if $S$ is a (measurable) region of space, then the probability that the particle will be found in $S$ is $\int_{S} \psi^{*} \psi=\int_{\mathbb{R}}\left(\chi_{S} \psi^{*}\right)\left(\chi_{S} \psi\right)$, where $\chi_{S}$ is 1 on $S$ and 0
} 
is something that is justified, in part, by appeal to the guidance equation. It would be going in a circle to then assume that the inner product has its usual physical significance when justifying the guidance equation. To conclude, I see no grounds on which Bohmians may appeal to Wigner's theorem at this point. And that means that Bohmians have no reason to think that boosts are represented by unitary operators.

Of course, the operators $e^{i m v x / \hbar}$ are certainly formally eligible to represent boosts in one dimension. (And there is a larger collection of operators containing them that forms a projective representation of the Galilean group, so that collection is collectively eligible to represent the action of Galilean transformations.) But many other collection of operators are also formally eligible. The problem for Bohmians is, which of these formally eligible collections is the one that correctly tells us how boosts act on the wavefunction? The considerations we have just looked at cannot be used to single out the collection used in the orthodox theory from the others.

The second justification for (B) proceeds as follows. ${ }^{14}$ We begin by assuming that boosts are a symmetry of the theory. We further assume that this means that boosts preserve $|\langle\psi, \phi\rangle|$. Then we appeal to Wigner's theorem and Stone's theorem to conclude that boosts are represented by a one-parameter family of unitary operators of the form $\exp (i v G)$, where $G$ is some, as yet unknown, self-adjoint operator. Then we calculate the commutation relations between the self-adjoint generators of the other one-parameter subgroups of the Galilean group. Assuming that $Q$ represents position (and that the particle has zero spin), it can then be shown that $G=m Q / \hbar$ is the only solution to those commutation relations.

This justification avoids analogies with Hamiltonian mechanics, but has the same two defects the first justification had. This justification also assumes that boosts preserve $|\langle\psi, \phi\rangle|$ (an assumption needed in order to make use of Wigner's theorem), and that $Q$ represents position. But the second assumption is false in Bohmian mechanics, and there are no grounds (at this stage in the construction of elsewhere. The function $\chi_{S}$ is just a projection operator on our Hilbert space, so this integral is just the inner product $\left\langle\chi_{S} \psi, \chi_{S} \psi\right\rangle=\left\|\chi_{S} \phi\right\|^{2}$.

${ }^{14}$ See, for example, Jordan 1969 (chapter VII) or Ballentine 1998 (chapter 3) for more details. 
Bohmian mechanics) for thinking the first one true.

I have just looked at two ways to justify (B). Neither of them is available in Bohmian mechanics, because both of them require that we understand the wavefunction the way that the orthodox theory understands the wavefunction. And that is not how we are to understand the wavefunction in Bohmian mechanics.

There is another way that Dürr, Goldstein, and Zanghì might to try defend (B). It emerges from looking at what they say about (A). They give a justification for taking time reversal to act on the wavefunction by complex conjugation that is different from the kinds of justifications for (B) that I have been discussing. Dürr et al. say that time reversal acts by complex conjugation because that is the way it needs to act to make Schrödinger's equation time reversal invariant. They might say something similar with respect to (B): only if (B) is true is Schrödinger's equation Galilean invariant. ${ }^{15}$ What makes this justification for (B) different in kind from the earlier ones is that it appeals to the wavefunction's dynamics. Dynamics played no role in the earlier justifications.

What should we think of this justification for (A) and (B)? I said earlier that claims about how Galilean transformations act on the wavefunction need to be independently grounded in order to be of use in the symmetry argument. And by 'independently grounded' I meant justified without any appeal to the dynamics. The idea was that if these claims did not need to be independently grounded then lots of candidate guidance equation could be made Galilean invariant by selecting weird enough ways for Galilean transformations to act on the wavefunction.

The justification for (A) and (B) we are now considering does appeal to the dynamics, so (A) and (B) are not independently grounded as I have been using this term. But the justification appeals to a part of the dynamics (Schrödinger's equation) that is independent of the guidance equation. Why is this not enough independence for (A) and (B) to then constrain which candidate we select to be the guidance equation?

My answer is that I think a justification for (A) and (B) that appeals to Schrödinger's equation is sufficiently independent to be of use in the symmetry argument. But that does not mean that the justification offered succeeds in giving us good reason to ac-

\footnotetext{
${ }^{15}$ Holland $(1993,122-4)$ appears to give this justification for (B).
} 
cept (A) and (B). So my objection to the dynamical justification of (A) and (B) is similar to my objection to the earlier justifications of (B) I discussed: if they succeeded they would be of use in the symmetry argument; but they do not succeed.

Why does the justification that appeals to Schrödinger's equation fail? Recall that the justification is that (A) and (B) need to be true in order for Schrödinger's equation to be time reversal invariant and Galilean invariant. But it is a mistake to assert that the action of boosts or of time reversal on physical states is whatever it needs to be to make a given equation Galilean invariant or time reversal invariant. (An analogous claim about the time reversal invariance of electromagnetism is defended forcefully in Arntzenius and Greaves 2009.) Abstractly speaking, for almost any dynamical law (including a law that on its face does not look time reversal invariant at all) we can find some transformation of states, call it 'the time reversal transformation,' and thus have the law come out 'time reversal invariant' (in this sense). But which operation is the time reversal operation is not so up for grabs. To determine whether a given equation is time reversal invariant, we have to first identify, in some independent way, how time reversal (and other symmetries) act on states, and only then check whether the equation is invariant under those symmetries. And what is true here about time reversal is also true for boosts.

Let us pause for a second and see where we are. So far I have challenged several justifications for (B). But I have not given any positive arguments for or against (B). So we are not yet in a position to know whether the symmetry argument for the guidance equation can succeed - to know that we need to know whether $(B)$ is true. So what might a successful positive argument for (or against) (B) look like? Here is one way an argument might proceed. Remember my discussion of the symmetry argument for Newton's law of inertia. There it was easy to see how Galilean transformations act on particle states: claims about how they act follow more or less immediately from facts about what particles are. So if one wants to defend the symmetry argument for the guidance equation the analogous procedure is to start by saying something about the nature of the thing represented by the wavefunction. Then we can see if that sheds any light on how Galilean transformations act on it (on whether (B) is true). I turn to this topic in the next section. 


\section{Remarks on the Nature of the Wavefunction in Bohmian Mechanics}

Saying how Galilean transformations act on the wavefunction in Bohmian mechanics is so complicated in part because it is not clear how we are to interpret the wavefunction (the mathematical object) in Bohmian mechanics. But if we did know what the wavefunction represents, then (perhaps) we would be in a position to know how the wavefunction transforms under Galilean transformations. Then we would know whether (B) is true, and so know how to evaluate the symmetry argument. There are several views out there about how to interpret the wavefunction in Bohmian mechanics. Let us look at a couple of them.

According to John Bell, the wavefunction represents a physical field like the electromagnetic field, which we can call 'The Wavefunction' (though unlike the electromagnetic field The Wavefunction lives in configuration space, not physical space). In a well-known passage he writes:

Note that in this compound dynamical system the wave is supposed to be just as 'real' and 'objective' as say the fields of classical Maxwell theory....No one can understand this theory until he is willing to think of $\psi$ as a real objective field rather than just a 'probability amplitude.' (Bell 1987b, 128; italics in original)

Bohm, in his original paper on Bohmian mechanics, seems to endorse the same view:

we have effectively been led to regard the wave function of an individual electron as a mathematical representation of an objectively real field. This field exerts a force on the particle in a way that is analogous to, but not identical with, the way an electromagnetic field exerts a force on a charge. (Bohm 1952, 170)

On this interpretation of Bohmian mechanics, The Wavefunction (in a world with a single particle) is a physical field that is represented by a (complex) scalar field on $\mathbb{R}^{3}$ (in single particle worlds configuration space can be identified with physical space). How do Galilean transformations act on this field? For some transformations the answer is obvious, and is the same answer we get in the orthodox theory: 
translations by $\alpha$ transform $\psi(x, t)$ to $\psi(x-\alpha, t)$. What about boosts? From the claim that The Wavefunction is a real objective field nothing immediately follows about how it transforms under boosts. But since this field is represented by a scalar function the most natural suggestion is that it transforms like a scalar field. ${ }^{16}$ If this suggestion is right then boosts should not change The Wavefunction at $t=0$ at all. At $t=0$ boosts should only change time derivatives, and (in this theory) The Wavefunction is not the time derivative of anything. (Of course, in the orthodox theory the wavefunction is not defined as the time derivative of anything, but it does 'encode information' about a time derivative, because it represents particle velocities. So it is okay for boosts to act non-trivially on the wavefunction in the orthodox theory. But The Wavefunction in Bohmian mechanics does not encode information about a time derivative. $)^{17}$

If this line of reasoning is right, then it is not true that the way boosts act on the wavefunction in Bohmian mechanics is the same as the way they act in the orthodox theory. And, what is more, the symmetry argument for the guidance equations fails, because the guidance equation turns out not to be Galilean invariant.

However, I do not here want to endorse this argument that Bohmian mechanics is not Galilean invariant. For the argument only works if the interpretation of the wavefunction in Bohmian mechanics as an objective scalar field is the only interpretation, or at least is the best interpretation. ${ }^{18}$ But there are other interpretations

${ }^{16}$ Maudlin $(2007,3169)$ claims that The Wavefunction is an objectively real field that does not transform like a scalar field under boosts. But his grounds for this are that it cannot transform that way if the guidance equation is to be Galilean invariant. Obviously, this is of no help in the present context.

${ }^{17}$ David Albert uses similar premises to argue that time reversal should not change the magnetic field in classical electromagnetism; see Albert 2000, chapter 1 .

${ }^{18}$ The argument here that Bohmian mechanics is not Galilean invariant rests on an assumption about how the wavefunction is to be interpreted. One might try to construct an argument that Bohmian mechanics is not Galilean invariant that does not rest on any assumptions like that. The argument in Valentini 1997 that I mentioned above is an argument of this form. Since my primary interest here is in what the wavefunction represents in Bohmian mechanics, I will not pursue this kind of argument here. 
out there.

For example, Dürr, Goldstein, and Zanghì (1997) present an alternative to the Bohm-Bell view that the wavefunction represents a 'real objective field'. They suggest instead that the wavefunction is an aspect of a physical law. As far as I understand this view, it says that the symbol ' $\psi$ ' as it appears in the laws of Bohmian mechanics does not even purport to represent some piece of ontology at all.

Perhaps there is a way, on this interpretation, to justify the claim that boosts act on the wavefunction just as they do in the orthodox interpretation. Obviously this interpretation would have to be filled in at a greater level of detail before we could evaluate any attempted justification. But if it could be done then the symmetry argument could be made to work and Bohmian mechanics could be shown to be Galilean invariant. I, however, do not know how to go about figuring out how boosts and other Galilean transformations act on the wavefunction on this interpretation (without, that is, appealing to the very equation we are trying to justify ${ }^{19}$ ).

To wrap up this section: I set out to look at what the wavefunction represents in Bohmian mechanics, hoping that that would shed some light on whether (A) and (B) are true. But the first view I looked at (The Bohm-Bell view) seems to imply that $(\mathrm{B})$ is false and the second does not have clear implications for the truth or falsity of (A) and (B).

\section{Conclusion}

There is excellent reason to take Bohmian mechanics seriously. It solves the measurement problem and accounts for all the phenomena that the orthodox theory accounts for. Still, there is important work out there for a justification of the guidance equation to do. It could help solve the underdetermination problem for Bohmian mechanics.

I have not tried to argue that no justification of the guidance equation can do this work. But I have argued that the symmetry argument for the guidance equation

\footnotetext{
${ }^{19}$ Allori et al. 2008 defend this interpretation of the wavefunction and then appeal to the guidance equation to justify the transformation properties of the wavefunction. Of course, once again, if one proceeds in this way then one cannot turn around and give the symmetry argument for the guidance equation.
} 
cannot. That argument relies on assumptions about how Galilean transformations act on the wavefunction which are correct in orthodox quantum mechanics. But I have not been able to find a way to justify those assumptions in Bohmian mechanics (in a way that does not already presuppose that the guidance equation is correct).

There is another moral that I would like to draw from the fact that within Bohmian mechanics we cannot arrive at the action of Galilean transformations on the wavefunction in the same way the orthodox theory does. The (absolute value of the) inner product on the Hilbert space $H$ in which the wavefunction lives has a clear physical meaning in orthodox quantum mechanics, ${ }^{20}$ and its having this meaning is a fundamental postulate of the theory (and of other collapse theories, like GRW). So the action of any symmetry of those theories on $H$ must preserve (the absolute value of) the inner product. But the physical meaning of the inner product on $H$ in theories in which the wavefunction never collapses is, at best, derived from (or at least less fundamental than) the fundamental postulates of the theory. (And there is plenty of dispute about whether these theories succeed at all in securing for the inner product the right physical significance.) These theories include Bohmian mechanics, and also Everettian interpretations of quantum mechanics. We need to be wary of the use in those theories of mathematical results (including, among other things, Wigner's theorem) whose standard application presupposes that the inner product gets its usual physical significance in the usual way.

\section{Acknowledgements}

Thanks to two anonymous referees, to Bryan Roberts, and to David Baker, Gordon Belot, Laura Ruetsche, and everyone else at the University of Michigan who read this paper.

\section{References}

Albert, David Z. 2000. Time and chance. Cambridge, MA: Harvard University Press.

\footnotetext{
${ }^{20}$ Insofar as the orthodox theory itself has a clear meaning, of course.
} 
Allori, Valia, Sheldon Goldstein, Roderich Tumulka, and Nina Zanghì. 2008. On the common structure of Bohmian mechanics and the Ghirardi-Rimini-Weber theory. British Journal for the Philosophy of Science 59: 353-389.

Arntzenius, Frank, and Hilary Greaves. 2009. Time reversal in classical electromagnetism. British Journal for the Philosophy of Science 60: 557-584.

Ballentine, Leslie. 1998. Quantum mechanics: A modern development. Hackensack, NJ: World Scientific.

Bell, John S. 1987a. On the impossible pilot wave. In Speakable and unspeakable in quantum mechanics, 159-168. New York: Cambridge University Press.

- 1987b. Quantum mechanics for cosmologists. In Speakable and unspeakable in quantum mechanics, 117-138. New York: Cambridge University Press.

Bohm, David. 1952. A suggested interpretation of the quantum theory in terms of ‘hidden' variables. I. Physical Review 85: 166-179.

Deotto, E., and G. C. Ghirardi. 1998. Bohmian mechanics revisited. Foundations of Physics 28: 1-30.

Dürr, D., S. Goldstein, and N. Zanghì. 1992. Quantum equilibrium and the origin of absolute uncertainty. Journal of Statistical Physics 67: 843-907.

- 1996. Bohmian mechanics as the foundation of quantum mechanics. In J. T. Cushing, A. Fine, and S. Goldstein (eds), Bohmian mechanics and quantum theory: An appraisal. Dordrecht: Kluwer Academic Publishers.

- 1997. Bohmian mechanics and the meaning of the wavefunction. In Experimental metaphysics, volume 193 of Boston studies in the philosophy of science, 25-38. Dordrecht: Kluwer Academic Publishers.

Earman, John, and Michael Friedman. 1973. The meaning and status of Newton's law of inertia and the nature of gravitational forces. Philosophy of Science 40: 329-359. 
Holland, Peter. 1993. The quantum theory of motion. Cambridge: Cambridge University Press.

Jordan, Thomas. 1969. Linear operators for quantum mechanics. New York: John Wiley and Sons, Inc.

Maudlin, Tim. 2007. Completeness, supervenience and ontology. Journal of Physics A: Mathematical and Theoretical 40: 3151-4171.

Peruzzi, Giulio, and Alberto Rimini. 2000. Compoundation invariance and Bohmian mechanics. Foundations of Physics 30: 1445-1472.

Valentini, Antony. 1997. On galilean and lorentz invariance in pilot-wave dynamics. Physics Letters A 228: 215-222.

Wallace, David. 2008. The quantum measurement problem: state of play. In Dean Rickles (ed), The Ashgate Companion to Contemporary Philosophy of Physics, 16-98. Burlington, VT: Ashgate. 